\title{
Repolymerization of pyrolytic lignin for producing carbon fiber with improved properties
}

\author{
Wangda $\mathrm{Qu}^{1}$, Yuan Xue ${ }^{1}$, Yiwei Gao ${ }^{1}$, Marjorie Rover $^{2}$, Xianglan Bai $^{1 *}$ \\ 1. Department of Mechanical Engineering, Iowa State University, Ames, Iowa, USA, 50011 \\ 2. Bioeconomy Institute, Iowa State University, Ames, Iowa, USA, 50011
}

\begin{abstract}
Lignin is a promising precursor of low-cost carbon fibers. However, the mechanical properties of carbon fibers produced from melt-spinning of raw lignin are poor, restricted by the randomly cross-linked polymer structures of lignin. In the present study, carbon fibers were produced from lignin-derived phenolic oil. Pyrolytic lignin was isolated from pyrolysis oil of red oak and washed with toluene to remove volatile impurities. Upon repolymerizing with $0.5 \%$ of sulfuric acid, the toluene-washed pyrolytic lignin became solid with the glass transition temperature $\left(\mathrm{T}_{\mathrm{g}}\right)$ of $101{ }^{\circ} \mathrm{C}$ and the average molecular weight of $1267 \mathrm{Da}$. The repolymerized pyrolytic lignin was further processed into carbon fibers through melt-spinning, oxidative stabilization and carbonization at $1000{ }^{\circ} \mathrm{C}$. The average tensile strength and modulus of the fibers were $855 \mathrm{MPa}$ and $85 \mathrm{GPa}$, while the highest values of individual fibers were $1014 \mathrm{MPa}$ and $122 \mathrm{GPa}$, respectively. The present study suggests that the quality of the carbon fiber produced from pyrolytic lignin could be further improved by process optimization.
\end{abstract}

Key words: fast pyrolysis, pyrolytic lignin, repolymerization, carbon fiber, acid catalyst

\footnotetext{
* Corresponding author. Tel: +1 515294 6886. Fax: +1 515294 3261. E-mail: bx19801@iastate.edu (Xianglan Bai)
} 


\section{Introduction}

Carbon fiber is a light weight material with superior mechanical properties and corrosion resistance. Thus, carbon fiber can be used as reinforced composites in automobile and aerospace industries, construction, sports equipment and more [1-3]. Despite great potential, the application of carbon fibers is currently limited, mainly due to the high cost of the precursor. Currently, a large portion of carbon fibers in the market are produced from polyacrylonitrile (PAN), and the precursor alone costs as much as $51 \%$ of the production cost of the carbon fiber [2]. PAN is derived from petroleum and releases toxic gas during carbon fiber processing, raising various environmental concerns $[4,5]$. Low-cost carbon fibers made from non-petroleum precursors could enable the application of carbon fibers in large scale. The Department of Energy suggested that the alternative carbon fibers with a tensile strength of $1720 \mathrm{MPa}$ and modulus of $172 \mathrm{GPa}$, and with cost below 11-15.40 $\$ \mathrm{~kg}^{-1}$, could be widely used in the automobile industry to reduce vehicle weight and improve fuel economy [6,7]. Lignin derived from lignocellulosic biomass has been of particular interest as an alternative precursor since it naturally contains a sixmembered-ring aromatic structure and is abundantly available at low cost $[8,9]$.

Lignin-based carbon fibers have been studied for nearly 50 years. The historical background of the development and recent advances have been thoroughly reviewed by Baker [7]. In the previous studies, carbon fibers were produced from lignin either by thermal processing, chemical modification or mixing lignin with other polymers. Although various types of lignin have been tested and the fiber manufacturing process was also carefully optimized, the quality of ligninbased carbon fibers is still too low to be considered for structural applications [8, 10-12]. To date, the lignin-derived carbon fiber with the highest quality was reported by Oak Ridge National Lab [13] with tensile strength and modulus of $1070 \mathrm{MPa}$ and $83 \mathrm{GPa}$, respectively. The fibers were made by melt-spinning an organic-solvent purified, Kraft hardwood lignin with a low glass transition temperature $\left(\mathrm{T}_{\mathrm{g}}=86^{\circ} \mathrm{C}\right)$ after thermal treatment. In general, lignin-based carbon fibers have tensile strength lower than $500 \mathrm{MPa}$.

The quality of lignin-based carbon fibers is low, attributed to the intrinsic nature of lignin structure. Lignin structure is also affected by its biomass origin and isolation method [14, 15]. Lignin is a phenyl propane-based macropolymer and is linked with carbohydrates through 
covalent bonds in biomass. To separate lignin from the rest of biomass, chemical treatments involving salts, acids, and/or organic solvents are commonly employed. Technical lignin produced either from paper, pulping or cellulosic biorefineries usually contain considerable amounts of organic and inorganic impurities derived from the biomass plant, the extraction process, as well as, lignin handling and storage. These impurities have to be removed from the lignin prior to carbon fiber production through acid washing, which otherwise cause various defects within the fibers. A melt-spinning method is generally used to process lignin due to its low cost. When lignin is melt-spun at elevated temperatures, the cleavage of weak ether and ester bonds in the lignin polymer release volatile products and create void spaces within the fibers [4] ultimately reducing the mechanical strength of the fibers. More importantly, lignin is a threedimensional polymer formed by random cross linking of its three precursor monomers. Due to the lack of molecular orientation and linearity within the polymer, the resulting fibers have amorphous structures, corresponding to low mechanical strength of the fibers.

While technical lignin is polymeric material isolated from biomass by the removal of carbohydrates through chemical or biochemical process, pyrolytic lignin is depolymerized lignin produced by heat. When lignocellulosic biomass is rapidly heated to $450{ }^{\circ} \mathrm{C}$ to $600{ }^{\circ} \mathrm{C}$ in an inert environment and the pyrolysis vapor is quenched, up to $75 \%$ of biomass can be recovered as biooil. Due to its robust process and low capital cost, pyrolysis is one of the major liquefaction technologies of biomass. During pyrolysis, phenolic monomers and oligomers produced from lignin (i.e., pyrolytic lignin) are condensed and form a complex mixture along with carbohydrate-derived sugars, furans, acids, ketones and aldehydes in bio-oil. Although accounting for $20 \%$ to $30 \%$ of bio-oil [16], pyrolytic lignin is usually referred to as the lesspreferred product because it increases the viscosity of bio-oil during storage and could even cause phase separation [17]. This is because pyrolytic lignin is unstable and has a tendency for polymerization. On the other hand, pyrolytic lignin can be extracted from bio-oil by simple water-washing and upgraded independently for other value-added applications. Potential applications of pyrolytic lignin include bio-adhesive [18], resin [19], bioasphalt [20], solid fuel [21], as well as, carbon fiber [22]. For example, Sahaf et al. [23] studied rheological and tunable thermoplastic properties of pyrolytic lignin and reported that pyrolytic lignin could be considered for hot melt adhesive applications. On the other hand, pyrolytic lignin as the precursor of carbon 
fiber can potentially provide several advantages over lignin. While it has some structural similarities with lignin [24], pyrolytic lignin is made of smaller phenolic units with reactive functional groups, such as phenolic hydroxyl groups, carbonyl groups and vinyl groups [16]. Thus, pyrolytic lignin has the potential to be re-polymerized [25] into more desirable structures versus lignin. Compared to as-received technical lignin, pyrolytic lignin may contain fewer impurities because the majority of impurities in biomass remain in biochar instead of bio-oil when biomass is pyrolyzed.

In the present study, carbon fibers were produced from pyrolytic lignin derived from the fast pyrolysis of red oak. Pyrolytic lignin isolated from the heavy fraction of bio-oil was repolymerized with acid catalyst prior to spinning into fibers. Both the precursor and carbon fibers were characterized in this work.

\section{Experimental}

\subsection{Preparation of pyrolytic lignin}

Red oak (Quercus Rubra) was purchased from Wood Residues Solutions (Montello, WI). It was first ground by a mill cut, and then pyrolyzed in a pilot-scale, fluidized bed reactor at Iowa State University's BioCentury Research Farm. Pyrolysis temperature was $500{ }^{\circ} \mathrm{C}$ and bio-oil was collected in a five-stage bio-oil condensation system. Detailed reactor configuration and the composition of bio-oil collected in each condenser was previously reported elsewhere [26]. Biooil collected in the first condenser consisted of pyrolytic sugars and phenols. It was waterwashed three times to remove sugars. The remaining water-insoluble fraction was designated as pyrolytic lignin and used in this study.

\subsection{Pretreatment of pyrolytic lignin}

Pyrolytic lignin was subsequently washed with toluene in a mass ratio of $1: 1$ three times for 15 min and then centrifuged for $20 \mathrm{~min}$ to remove any residual water and toluene. The toluenewashed pyrolytic lignin was further heated at $90{ }^{\circ} \mathrm{C}$ for 15 min to remove any volatiles. Each time, $10 \mathrm{~g}$ to $15 \mathrm{~g}$ of toluene-washed pyrolytic lignin was placed in a $200 \mathrm{ml}$ beaker and set on a heating plate. A funnel with its cone-side downward was placed right above the beaker. The neck-side of the funnel was connected to a vacuum tube to facilitate the removal of volatiles. For 
thermal treatment, the pyrolytic lignin was heated in an oil bath at $105{ }^{\circ} \mathrm{C}$ for $2 \mathrm{~h}$ with a magnetic stirring bar. A $0.5 \%$ of sulfuric acid (SA) was added dropwise into the pyrolytic lignin to catalyze polymerization reaction [27, 28]. The produced precursor was designated as SA treated precursor in this study.

\subsection{Characterization of pyrolytic lignin}

Dionex Ultimate 3000 series high performance liquid chromatography (HPLC) together with a Shodex Refractive Index (RI) and Diode Array Detectors (DAD) was used to conduct gel permeation chromatography (GPC) analyses for relative molecular weight distribution. Two GPC columns $(3 \mu \mathrm{m}, 100 \AA$, $300 \times 7.5$ mm; PLgel, Agilent, p/n PL1110-6320) were calibrated with six monodispersed polystyrene standards ranging from $162 \mathrm{~g} \mathrm{~mol}^{-1}$ to $38640 \mathrm{~g} \mathrm{~mol}^{-1}$. Tetrahydrofuran was used as the solvent for samples and the eluent in the columns.

An Agilent 7890B gas chromatography (GC) with Agilent 5977A mass-selective-detector (MSD) and flame ionization detector (FID) system was used to identify the chemical composition in the pyrolytic lignin and precursors. The capillary column used in the GC was a ZB-1701 (60 $\mathrm{m} \times$ $250 \mu \mathrm{m} \times 0.25 \mu \mathrm{m})$. The injection temperature was $250^{\circ} \mathrm{C}$ and the oven temperature was kept at $40{ }^{\circ} \mathrm{C}$ for $3 \mathrm{~min}$, and then ramped to $280{ }^{\circ} \mathrm{C}$ with $3{ }^{\circ} \mathrm{C} \mathrm{min}^{-1}$.

A Thermo Scientific Nicolet iS10 (Thermo Fisher Scientific Inc., Waltham, MA) equipped with a Smart iTR accessory was used to conduct Fourier Transform Infrared (FTIR) analysis to determine the functional groups in the pyrolytic lignin and precursors. With wave numbers ranging from $750 \mathrm{~cm}^{-1}$ to $4000 \mathrm{~cm}^{-1}$, each sample was scanned 32 times at a resolution of $4 \mathrm{~cm}^{-1}$ and interval of $1 \mathrm{~cm}^{-1}$.

Thermogravimetric analysis (TGA) was conducted using a Mettler Toledo TGA/DSC instrument. The samples were heated at a rate of $10{ }^{\circ} \mathrm{C} \mathrm{min}-1$ from $25{ }^{\circ} \mathrm{C}$ to $1000{ }^{\circ} \mathrm{C}$ under nitrogen with a flow rate of $100 \mathrm{ml} \mathrm{min}^{-1}$. The experiment was duplicated for reproducibility.

Glass transition temperature $\left(\mathrm{T}_{\mathrm{g}}\right)$ was determined by using a differential scanning calorimeter (DSC, Q20, TA instruments). Each sample was heated to $125{ }^{\circ} \mathrm{C}$ with a heating rate of $10{ }^{\circ} \mathrm{C}$ $\min ^{-1}$. Nitrogen was used as the purge gas with a flow rate of $50 \mathrm{ml} \mathrm{min}{ }^{-1}$. The midpoint $\mathrm{Tg}$ of precursor was determined by using a TA software. 
The CHNS elemental analysis of the pyrolytic lignin and precursor were conducted using Elementar (vario MICRO cube) elemental analyzer.

\subsection{Production of carbon fiber}

About $6 \mathrm{~g}$ of pyrolytic lignin was fed into a twin-screw microcompounder (DACA Instruments, Santa Barbara, CA) and extruded at $115{ }^{\circ} \mathrm{C}$ to $120{ }^{\circ} \mathrm{C}$. The fibers were wound onto a roller (DSM, Geleen, Netherlands) at up to $100 \mathrm{~m} \mathrm{~min}^{-1}$. The oxidative stabilization of the spun fibers were conducted in a muffle furnace by heating the fibers up to $280{ }^{\circ} \mathrm{C}$ at a heating rate of $0.3{ }^{\circ} \mathrm{C}$ $\min ^{-1}$ and then held for $1 \mathrm{~h}$. Carbonization was performed in a tubular furnace under an argon environment. The stabilized fibers were heated at $3{ }^{\circ} \mathrm{C} \min ^{-1}$ up to $1000{ }^{\circ} \mathrm{C}$, and held for $1 \mathrm{~h}$.

\subsection{Characterization of carbon fibers}

Tensile strength of single fiber was measured using a dynamic mechanical analyzer (DMA) (Q800, TA Instruments), the stress-strain curve was obtained by stretching fiber at $30{ }^{\circ} \mathrm{C}$ under a strain rate of $50 \mu \mathrm{m} \mathrm{min}^{-1}$ [2]. The diameter of each fiber was measured by an electronic digital micrometer with a resolution of $1 \mu \mathrm{m}$ and further confirmed with scanning electron microscope.

The microstructure of the carbon fiber samples was examined using a scanning electron microscope (SEM, Quanta-FEG 250, FEI) at $10 \mathrm{kV}$ accelerating voltage. Segments of the samples were mounted onto double-stick carbon tape on a $45^{\circ}$ incline. Samples were coated with $5 \mathrm{~nm}$ of iridium for conductivity.

\section{Results and discussion}

\subsection{Properties of pyrolytic lignin}

Pyrolytic lignin and pyrolytic lignin after toluene wash were a dark black, viscous liquid at room temperature. The viscosity of the pyrolytic lignin initially decreased at elevated temperatures but later increased as thermal treatment progressed. Upon cooling to room temperature, the SA treated pyrolytic lignin became solid.

\subsubsection{GPC analysis}


Relative weight average molecular weight (Mw) and polydispersity index (PDI) of the pyrolytic lignin before and after it was toluene-washed, and SA treated are listed in Table 1. The relative Mw distribution of the pyrolytic lignin treated differently is also given in Figure 1. As shown, the pyrolytic lignin before the toluene wash had a relative Mw of $566 \mathrm{Da}$ and PDI of 2.44. In the GPC chromatogram, the relative $\mathrm{Mw}$ of the major peak was $158 \mathrm{Da}$ while two minor peaks appeared at $84 \mathrm{Da}$ and $452 \mathrm{Da}$, respectively. Since the theoretical $\mathrm{Mw}$ of a phenolic unit is around $180 \mathrm{Da}$ [29], the pyrolytic lignin is a mixture of phenolic monomers and oligomers. The phenolic monomers usually have a lower viscosity than oligomers. The precursor with too low of a viscosity is difficult to spin into continuous fibers, and easily creating void spaces within the fibers due to its volatility during the melt spinning process. Therefore, the pyrolytic lignin was toluene-washed to remove low $\mathrm{Mw}$ compounds. The non-polar solvent fraction also preferentially removes less reactive compounds with lower degree of polymerization [30]. After toluene washing, the content of phenolic monomers with relative Mw less than $200 \mathrm{Da}$ were substantially decreased and the Mw of the pyrolytic lignin increased to $810 \mathrm{Da}$. The PDI value also decreased slightly, indicating that the toluene-washed pyrolytic lignin had a narrower Mw distribution after washing. Baker et al. [7] suggested that lignin with a narrower Mw distribution was more desirable as a precursor.

The addition of a small amount of sulfuric acid (SA) during thermal treatment increased the Mw of the pyrolytic lignin to $1267 \mathrm{Da}$. Acid catalyzes polymerization of lignin-derived phenolic compounds. For instance, carboxylic acids (acetic acid, formic acid etc.) in bio-oil are known to promote polymerization of phenols during bio-oil storage [28]. Due to its strong acidity, a small amount of SA was enough to increase $\mathrm{Mw}$ of the precursor at relatively short reaction time. A higher concentration of SA results in the precursor charring, making it unable to be melt-spun. The corresponding Mw distribution of the pyrolytic lignin showed a significant decrease in low Mw phenols, accompanied by the formation of higher Mw oligomers. The PDI value was found to increase from 2.42 before treatment to 2.73 after treatment. The polydispersity of SA treated precursor increased slightly, suggesting that the reactivity of the functionalities in phenolic oligomers are varied, which resulted in a wider range of molecular distribution after polymerization. Sulfuric acid is an effective catalyst for polymerization reaction and it could 
protonate carbonyl, vinyl and hydroxyl groups to promote the overall reactivity of pyrolytic lignin.

Table 1. Relative weight average molecular weight (Mw) and polydispersity of pyrolytic lignin before and after treatments.

\begin{tabular}{lcccc}
\hline Pyrolytic lignin & Mw (Da) & Mn (Da) & Polydispersity & $\begin{array}{c}\text { Total intensity percentage (\%) } \\
\text { (Molecular weight < 200 Da) }\end{array}$ \\
Without toluene wash & 566 & 232 & 2.44 & 37.1 \\
After toluene wash & 810 & 335 & 2.42 & 16.3 \\
SA treated & 1267 & 464 & 2.73 & 11.9 \\
\hline
\end{tabular}

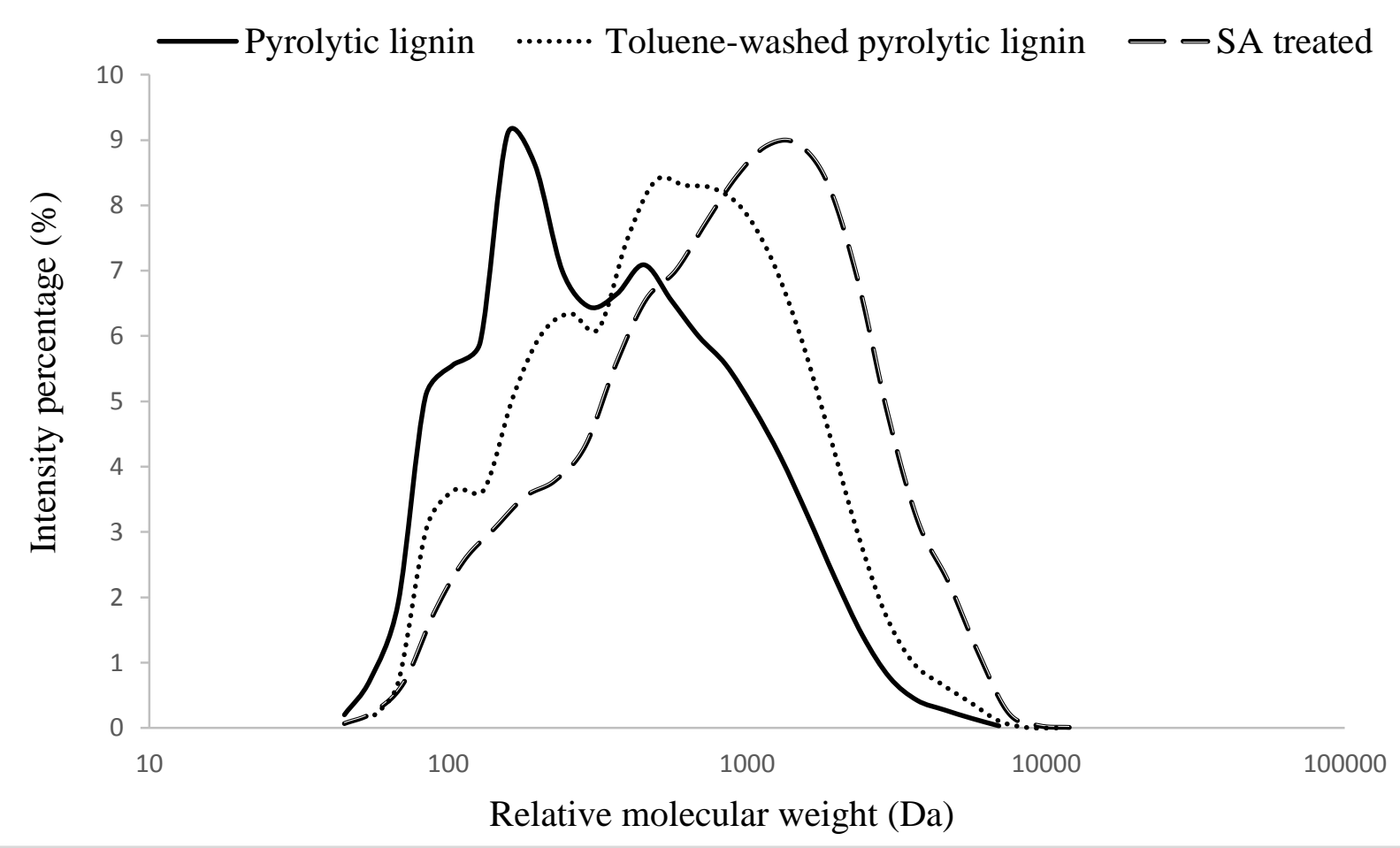

Figure 1. Relative molecular weight distribution of pyrolytic lignin before and after pretreatment.

\subsubsection{GC/MS analysis}

The compositions of the pyrolytic lignins with different treatments are compared in Figure 2. Phenolic oligomers are not shown in the chromatograms due to their low volatility in the GC 
column. The major phenolic monomers in the water-washed pyrolytic lignin include syringol, 1,2,4-trimethoxybenzene, 3',5'-dimethoxyacetophenone, 2,6-dimethoxy-4-(2-propenyl)-phenol, etc. Carbohydrate-derived organics, such as 1,2-cyclopentanedione, 2-cyclopenten-1-one, and levoglucosan were also found in the residues. After the toluene wash, peak intensities of the carbohydrate-derived organics and the majority of phenolic monomers decreased. However, the peak intensities of phenolic monomers that appear at retention times greater than $61 \mathrm{~min}$ did not change much. The phenolic monomers with higher retention times have longer side chains and larger Mw. Apparently, the phenolic monomers with lower Mw have better solubility in toluene versus the higher $\mathrm{Mw}$ phenols. After acid-catalyzed polymerization, an overall decrease in the peak intensities of the compounds, regardless of their retention times, was observed. Particularly, the phenols with side chain $\mathrm{C}=\mathrm{C}$ and $\mathrm{C}=\mathrm{O}$ virtually disappeared, because these functional groups have a higher tendency for polymerization. 

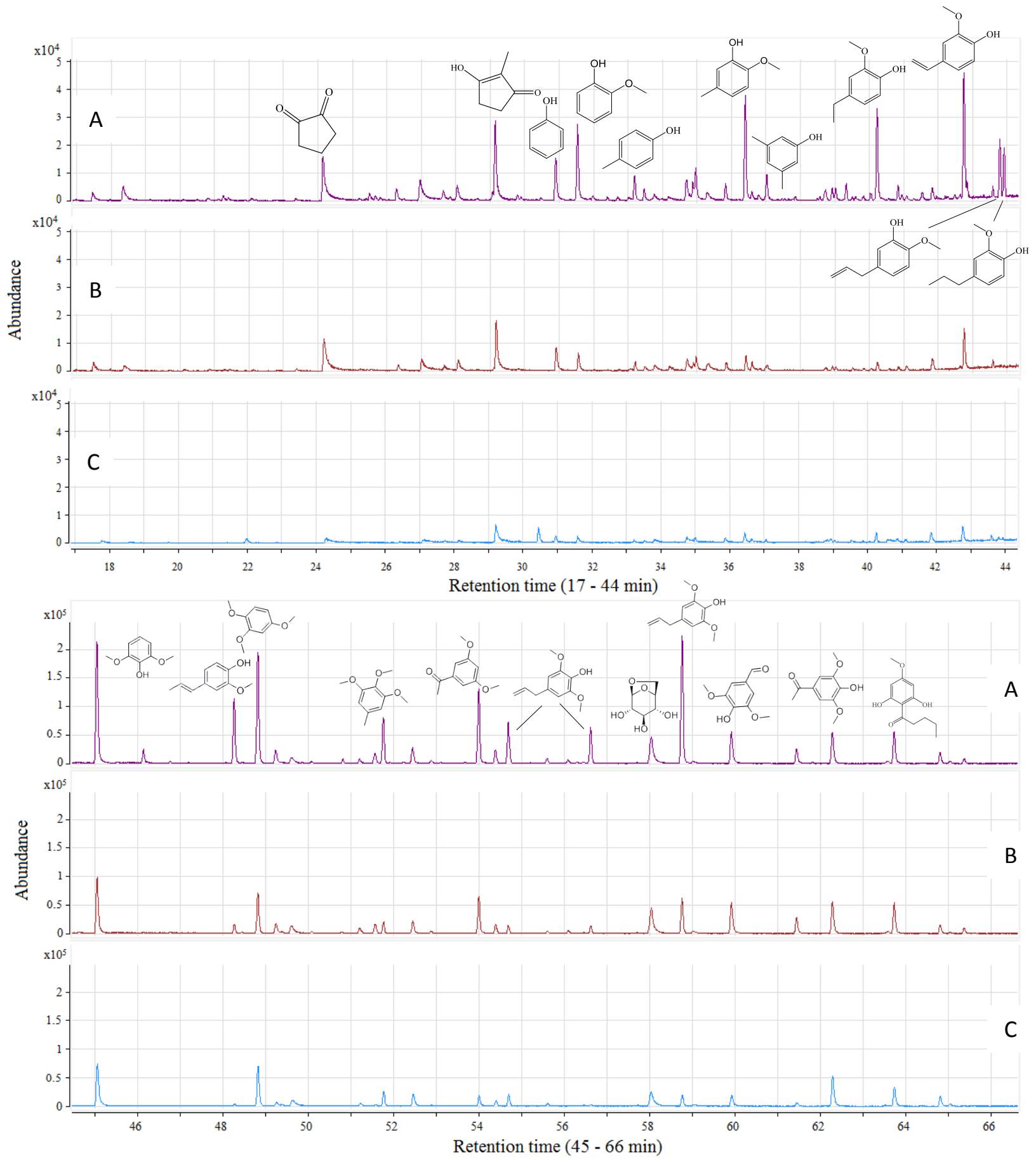

Figure 2. GC/MS chromatogram of pyrolytic lignin: A) Pyrolytic lignin; B) Toluene-washed pyrolytic lignin; C) SA treated precursor; Top: retention time (17-44 min); Bottom: retention time (45-66 min). 


\subsubsection{FTIR analysis}

FTIR spectra of the pyrolytic lignin were also compared in Figure 3. The different peaks were normalized using the peak that appears at $1510 \mathrm{~cm}^{-1}$, which represents aromatic-skeleton vibration. The peak at $3368 \mathrm{~cm}^{-1}$, corresponding to an $\mathrm{OH}$ stretch, was the highest in waterwashed pyrolytic lignin. It could be related to phenolic $\mathrm{OH}$ and residual water remaining in the sample. The peak was reduced in the toluene-washed pyrolytic lignin, due to the removal of water and some phenols. The remainders of the peaks in the FTIR spectra were similar for waterwashed and toluene-washed pyrolytic lignin. The SA treatment decreased the $\mathrm{OH}$ band significantly, indicating that polymerization occurred through phenolic $\mathrm{OH}$ sites. The intensities of other IR bands also changed significantly SA treatment. The band appearing at $1700 \mathrm{~cm}^{-1}$, represents conjugated aldehydes and carboxylic acid, decreased after the treatment since carbonyl groups are highly reactive. The blunt band appearing around $1634 \mathrm{~cm}^{-1}$ for both the water-washed and toluene-washed pyrolytic lignin is related to levoglucosan and other carbohydrate-derived residue compounds. It nearly disappeared after SA treatment. The band at $1602 \mathrm{~cm}^{-1}$ corresponds to aromatic skeletal vibrations plus $\mathrm{C}=\mathrm{O}$ stretch also decreased due to the reactivity of $\mathrm{C}=\mathrm{O}$ bond for polymerization.

The bands that appear at $1500 \mathrm{~cm}^{-1}$ or below are related to different bond stretches associated with phenolic aromatic structures. For example, the band at $1200 \mathrm{~cm}^{-1}$ corresponds to C-C, C-O, and $\mathrm{C}=\mathrm{O}$ stretch in the guaiacol structure; the bands at $1104 \mathrm{~cm}^{-1}$ and $1035 \mathrm{~cm}^{-1}$ represent aromatic $\mathrm{C}-\mathrm{H}$ in plane deformation. Distinguishing these bands from each other became more difficult after the SA treatment, suggesting that the character of the phenolic structure disappears and a more condensed polyaromatic structure is formed. Previously, we noted that the aforementioned bands disappear in pyrolysis char of lignin due to its high polyaromatic structure [31]. The band that appears at $958 \mathrm{~cm}^{-1}$ related to $-\mathrm{HC}=\mathrm{CH}$ out-of-plane deformation decreased, possibly due to the polymerization of vinyl or propyl bonds. Interestingly, the band that appears at $2842 \mathrm{~cm}^{-1}$ did not change significantly during the SA treatment. This suggests that demethoxylation or demethylation was negligible during polymerization of the pyrolytic lignin. 


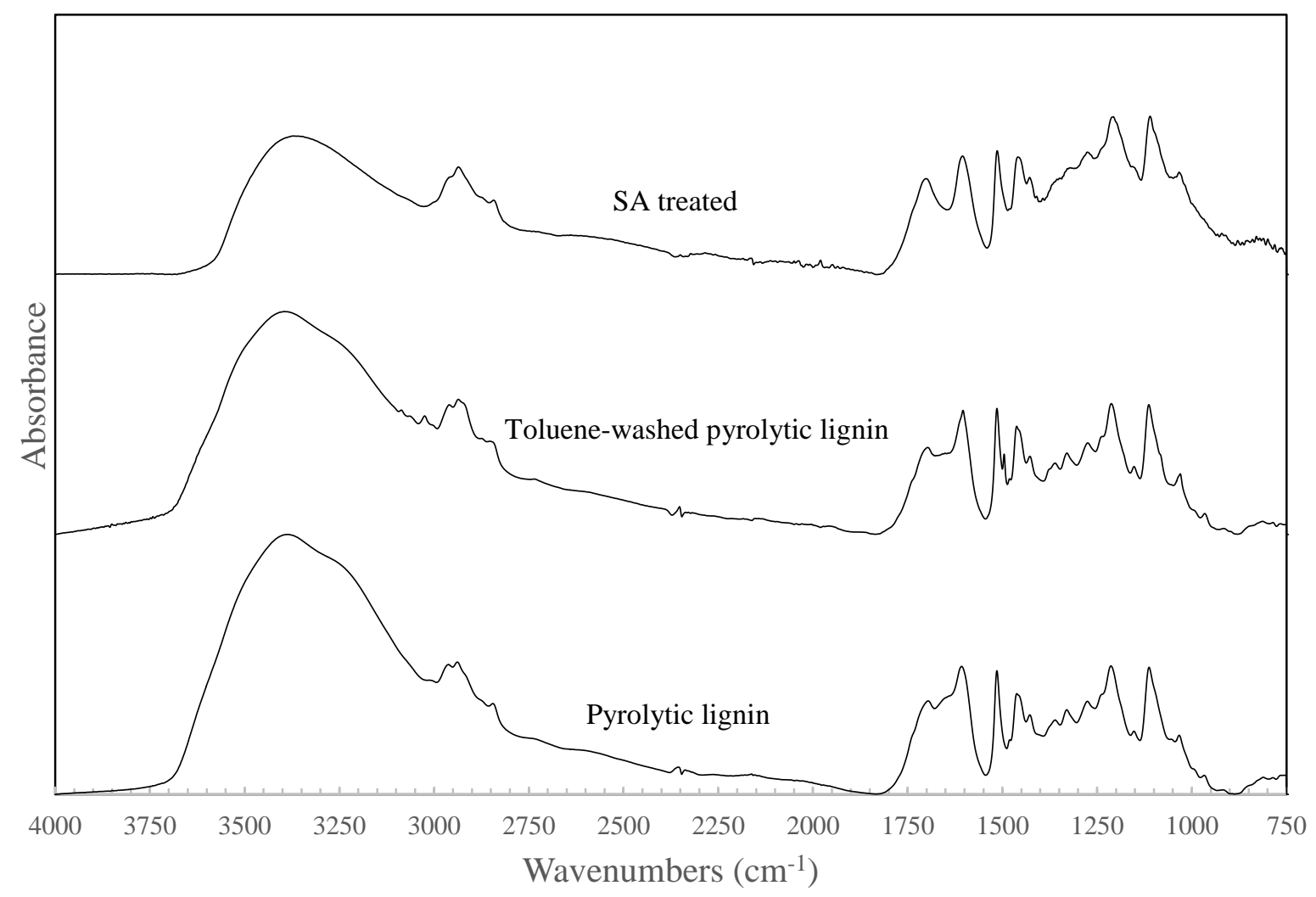

Figure 3. FTIR spectra of pyrolytic lignin before and after pretreatment.

\subsubsection{Thermal stability}

Thermal stability of the pyrolytic lignin was investigated using thermogravimetric analysis (TGA) and the TGA profiles are compared in Figure 4. The mass loss of toluene-washed pyrolytic lignin began below $100{ }^{\circ} \mathrm{C}$, probably due to incomplete removal of water, toluene and other low molecular weights products remaining in the pyrolytic lignin. In comparison, the SA treatments greatly improved the thermal stability of the pyrolytic lignin. The major mass loss did not begin until the temperature was above $150{ }^{\circ} \mathrm{C}$ (mass loss $<1 \%$ ). The SA-treated pyrolytic lignin had a good thermal stability and the higher yield of mass residue (i.e., 35\%) at the end of the heating, which corresponds to the results obtained from GPC and FTIR, indicating it contained compounds with higher Mw and more condensed structure. 


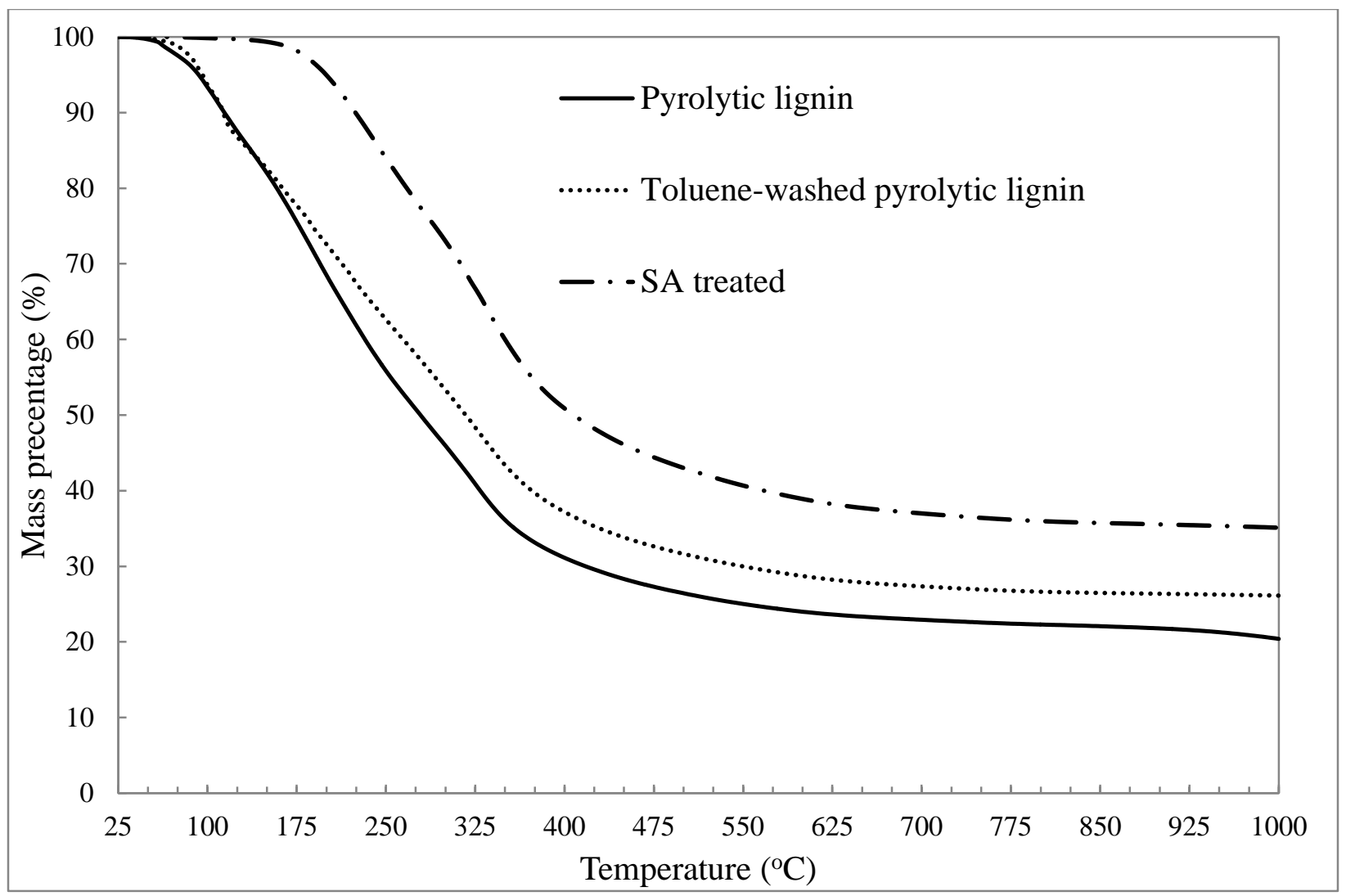

Figure 4. TGA profiles of toluene-washed pyrolytic lignin and SA treated precursor.

\subsubsection{Elemental composition of pyrolytic lignin}

The elemental analysis of the pyrolytic lignin before and after treatment is given in Table 2 . The carbon content in pyrolytic lignin before the toluene wash was $54.84 \%$ and the oxygen content was $38.52 \%$. After the toluene wash, carbon content increased to $61.73 \%$ and the oxygen content decreased to $31.34 \%$ since the residual water was removed. After the SA treatment, the carbon content further increased to $64.35 \%$, whereas, oxygen content decreased. Hydrogen content also decreased, suggesting that condensation polymerization during the SA treatment possibly produced water as the byproduct, which was then evaporated. The concentration of sulfur increased since the sulfur would remain in the pyrolytic lignin after polymerization. 
Table 2. Elemental composition of pyrolytic lignin before and after pretreatments.

\begin{tabular}{lccccc}
\hline Pyrolytic lignin & $\mathrm{C}(\%)$ & $\mathrm{H}(\%)$ & $\mathrm{N}(\%)$ & $\mathrm{S}(\%)$ & $\mathrm{O}(\%)^{*}$ \\
Before toluene-wash & 54.84 & 5.89 & 0.74 & 0.01 & 38.52 \\
After toluene-wash & 61.73 & 6.21 & 0.71 & 0.01 & 31.34 \\
SA treated & 64.46 & 5.64 & 0.62 & 0.13 & 29.15 \\
\hline
\end{tabular}

* By difference.

\subsubsection{Differential Screening Calorimetry}

The DSC curves of the SA-treated pyrolytic lignin were measured and the results are shown in Figure 4. The sharp peak on the DSC curve, as the indicator of glass transition temperature $\left(\mathrm{T}_{\mathrm{g}}\right)$, was not found. Instead, the DSC curve of the pyrolytic lignin was relatively smooth over the course of measurement. This is due to the nature of pyrolytic lignin, which is a mixture of various phenolic compounds. However, small dip was identified on the curve and the $\mathrm{T}_{\mathrm{g}}$ could be assigned, which was $101{ }^{\circ} \mathrm{C}$. It is known that hardwood lignin usually has a lower $\mathrm{T}_{\mathrm{g}}$ than the lignin originating from other biomass species [32], and this could also apply to pyrolytic lignin derived from hardwood lignin. The degree of cross-linking and Mw could impact $\mathrm{T}_{\mathrm{g}}$ of the materials. Increased $\mathrm{Mw}$ and more condensed aromatic structure in the SA-treated pyrolytic lignin are likely responsible for the $\mathrm{T}_{\mathrm{g}}$. 


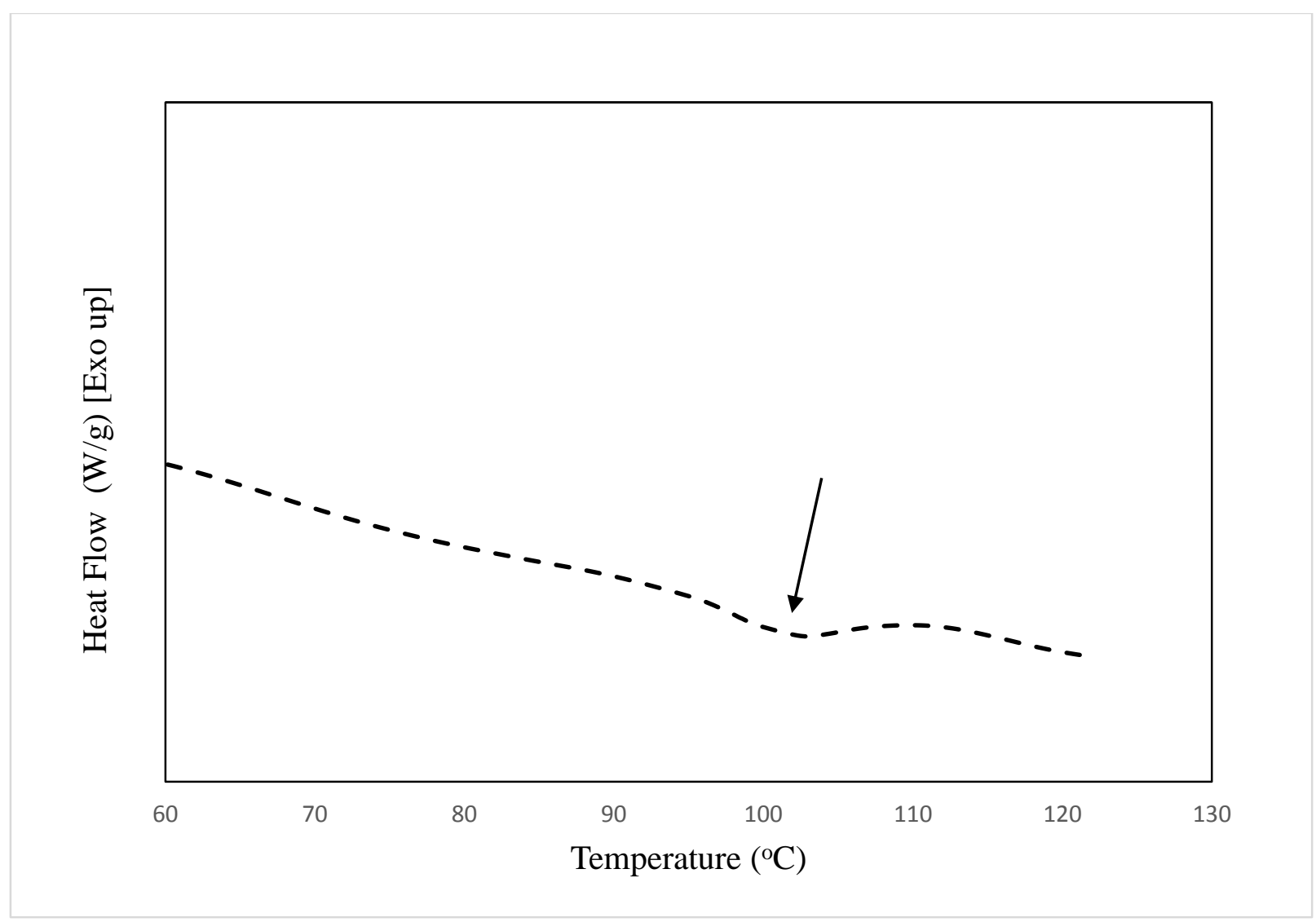

Figure 5. DSC profiles of the SA treated precursor $\left(\mathrm{T}_{\mathrm{g}}=101^{\circ} \mathrm{C}\right)$.

\subsection{Carbon fiber processing}

The water-washed and toluene-washed pyrolytic lignins were highly viscous at room temperature. Increasing temperature to $80{ }^{\circ} \mathrm{C}$ reduced the viscosity of the liquid sufficiently allowing fibers to be spun continuously. However, the spun fibers fused together upon cooling to room temperature. This was due to the low $\mathrm{Mw}$ and $\mathrm{T}_{\mathrm{g}}$ of the precursors. By further increasing the spinning temperature, the viscosity of the pyrolytic lignins reduced, making them unable to form a continuous fiber. In comparison, the SA treated pyrolytic lignin was able to spin into fibers continuously upon heating. The fiber-spinning temperature was determined based on the TGA and DSC results previously shown. A spinning temperature higher than its softening point is preferred, while high temperature could cause decomposition and volatilization of the precursor. Therefore, the spinning temperature was initially set at $115^{\circ} \mathrm{C}$ for the SA treated pyrolytic lignin. Based on the TGA analyses, the mass loss of the precursor at the temperature was negligible and no volatile formation was expected during the fiber spinning process. 
The spun fibers were subjected to oxidative thermal stabilization. Stabilization is a critical step in determining the overall properties of carbon fibers. The fiber is converted from a thermoplastic material into a thermoset material after stabilization. Usually, a low heating rate is required to assure adequate oxidation, and cross-linking among inter and intra molecules occurs with increasing temperature. The $\mathrm{T}_{\mathrm{g}}$ of the fiber needs to be higher than the stabilization temperature in the course to retain the rigidity of the fiber and prevent thermal fusion. Tension was not applied to the fiber during stabilization. However, it was noticed that the fibers that vertically hung on the metal rack stretched during the stabilization due to gravity. After stabilization, the stabilized fibers become stronger and more flexible than the as-spun fibers.

Carbonization removes most of the other organic elements in the fibers and increases carbon content. A condensed polyaromatic structure is also formed during carbonization. $\mathrm{CHN}$ analysis indicated that the carbon content exceeded $93 \%$ after carbonizing the fibers at $1000{ }^{\circ} \mathrm{C}$.

\subsection{Characterization of carbon fiber and discussion}

As shown in Table 3, the average tensile strength and modulus of the carbon fibers produced from the SA-treated pyrolytic lignin were $855 \mathrm{MPa}$ and $85 \mathrm{GPa}$, respectively. The maximum values of individual fiber reached a tensile strength of $1014 \mathrm{MPa}$ and modulus of $122 \mathrm{GPa}$. The average diameter of the fibers was $36 \mu \mathrm{m}$. SEM images of the carbon fibers produced from the SA treated pyrolytic lignin are shown in Figure 6. It could be observed that carbon fiber produced from pyrolytic lignin based precursor in this study is smooth and solid.
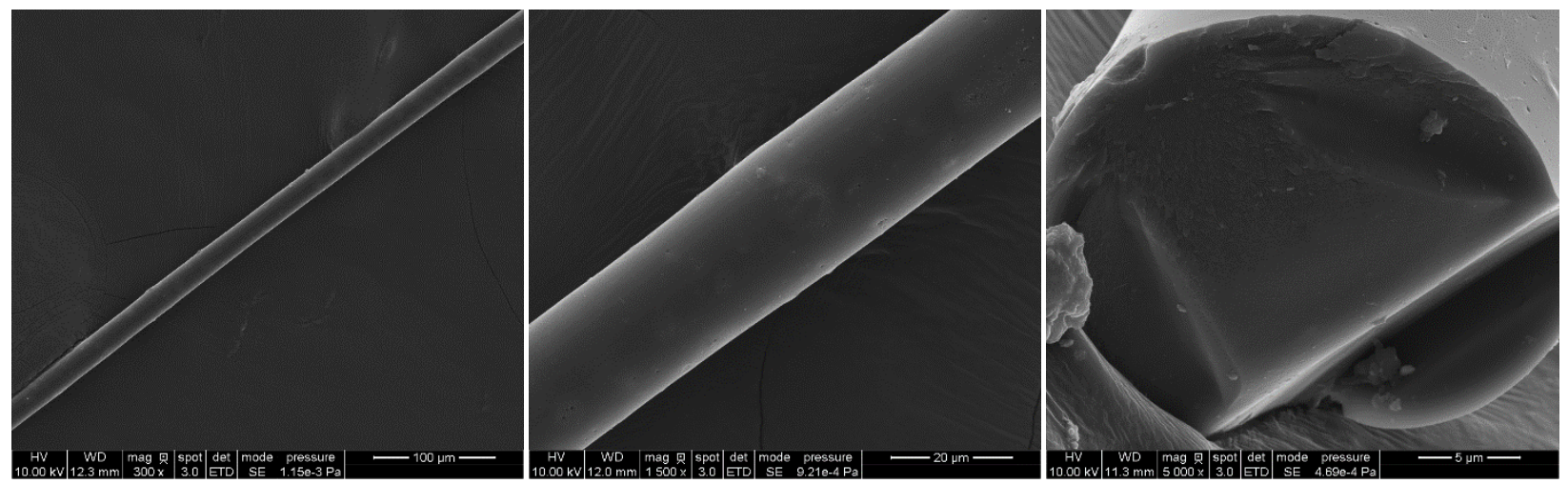

Figure 6. SEM images of carbon fibers produced from SA treated precursor 
In the present study, carbon fibers were made from pyrolytic lignin by the repolymerization of lignin-derived phenolic monomers and oligomers. However, repolymerization of the depolymerized lignin does not reversibly form lignin. During fast pyrolysis, a high level of thermal energy is provided to lignin within an extremely short time to promote rapid dissociation of chemical bonds. As a result, the ether and ester bonds that connecting aromatic ring units cleave and various chemical reactions occur. Not only a number of phenolic monomers and oligomers are formed, but $\mathrm{CO}_{2}, \mathrm{CO}, \mathrm{CH}_{4}$ and $\mathrm{H}_{2} \mathrm{O}$ are also released through decarboxylation, decarbonylation, demethylation and dehydrogenation [33]. The pyrolytic lignin is ready to repolymerize due to the reactivity, but rearrangement of the phenolics would form a modified polymer rather different from the original lignin [34].

Table 3 compares the properties of the carbon fibers produced in the present study with two types of lignin-based carbon fibers reported in literature. The reference carbon fibers are made from a hardwood lignin [7] or acetylated softwood lignin [35] through well-optimized processes, representing the highest quality a lignin-based carbon fiber can achieve, to date.

While the mechanical properties of the carbon fibers obtained from the SA treated pyrolytic lignin were nearly comparable to the two reference carbon fibers, the quality of the pyrolytic lignin-based carbon fibers could be further improved by optimizing the precursor and processing conditions.

Table. 3. Comparison of mechanical properties of carbon fibers produced in the present work with the best-quality lignin-based fibers from previous studies.

\begin{tabular}{lccccc}
\hline Sample & $\begin{array}{c}\text { Tensile strength } \\
(\mathrm{MPa})\end{array}$ & $\begin{array}{c}\text { Modulus } \\
(\mathrm{GPa})\end{array}$ & Strain $(\%)$ & $\begin{array}{c}\text { Diameter } \\
(\mu \mathrm{m})\end{array}$ & Tension \\
\hline SA treated & $855 \pm 159(1014) *$ & $85 \pm 37(122)$ & $1.01 \pm 0.3$ & $29-50$ & No \\
\hline Kraft hardwood [7] & 1070 & 83 & 1.29 & $<10$ & Yes \\
\hline \multirow{2}{*}{ Acetylated softwood [35] } & $510 \pm 50$ & $30 \pm 2$ & $1.7 \pm 0.1$ & 22.5 & No \\
& $1050 \pm 70$ & $35 \pm 3$ & $3.0 \pm 0.2$ & $5.9 \pm 0.2$ & Yes
\end{tabular}

*Values in parentheses stands for the highest value obtained among all tests. 
The diameters of the carbon fibers produced in the present study were significantly larger than that of the reference fibers which are below $10 \mu \mathrm{m}$. It is known that the tensile strength of the fiber is highly dependent of fiber diameter [36]. The thinner the fiber obtained, the higher tensile strength it will retain. Improving the sheer force of the fibers during the spinning process by using a smaller sized die and higher fiber spinning rate could reduce the fiber diameter. Applying tension during stabilization and carbonization can also reduce the diameter of the fibers. Applying tension would also improve the orientation of the fiber molecules, therefore, increasing the mechanical property of the fibers [37]. Meng and Ogale [38] reported the length of the acetylated softwood lignin fibers stretched as much as 8 times when tension was applied during stabilization process. By applying tension during both stabilization and carbonization, they were able to produce the carbon fibers with diameter of $7 \mu \mathrm{m}$, a tensile strength of $1010 \mathrm{MPa}$ and modulus of $53 \mathrm{GPa}$. Without tension, the tensile strength of the carbon fiber was $530 \mathrm{MPa}$.

While stabilization time depends on the $\mathrm{T}_{\mathrm{g}}$ of the fibers, the $\mathrm{Mw}$ of the pyrolytic lignin and $\mathrm{T}_{\mathrm{g}}$ can be manipulated by adjusting the concentration of SA and/or pretreating temperature and time. The precursor fibers with higher $T_{g}$ can be stabilized in shorter times. However, too high $T_{g}$ is not preferred as it may produce a precursor that becomes difficult to spin into fiber.

Although, further optimization is still needed, the properties of the pyrolytic lignin based carbon fibers in the present study are comparably high. Pyrolytic lignin is structurally different from lignin. Wang et al. [39] previously reported that pyrolytic lignin contains newly formed unconjugated $\mathrm{C}-\mathrm{O}$ functional groups and a fewer amount of methoxyl groups in comparison to lignin. Therefore, repolymerizing the lignin-derived phenolic monomers and oligomers may have resulted in a modified polymer with an improved potential as the precursor in comparison to lignin.

\section{Conclusions}

Carbon fibers were made from pyrolytic lignin isolated from the heavy fraction of bio-oil. Pyrolytic lignin is a mixture of phenolic monomers and oligomers derived from the thermal decomposition of lignin. The pyrolytic lignin was first washed with toluene to remove low-Mw compounds and to narrow the Mw distribution. The pyrolytic lignin was repolymerized in the presence of an acid catalyst to increase both thermal stability and the $T_{g}$ of the precursor. After 
melt-spinning, oxidative stabilization and carbonization, the average tensile strength and modulus of the carbon fibers were $855 \mathrm{MPa}$ and $85 \mathrm{GPa}$, respectively. Individual carbon fiber obtained a tensile strength as high as $1014 \mathrm{MPa}$ and a modulus of $122 \mathrm{GPa}$. The results of the present study suggest that pyrolytic lignin is a promising precursor for the production of low-cost carbon fibers.

\section{Acknowledgements}

The funding support from National Science Foundation Center for Bioplastics and Biocomposites is greatly acknowledged. The bio-oil used in this study was provided by Prof. Robert Brown and produced by his students and staff at Biorenewable Research Farm of Iowa State University. The authors would also like to thank Melissa Montalbo-Lomboy, Patrick Johnston, Ryan Smith and Patrick Hall for technical support and Dr. Straszheim for assistance in SEM analysis.

\section{References}

[1] Frank E, Hermanutz F, Buchmeiser MR. Carbon fibers: precursors, manufacturing, and properties. Macromolecular materials and engineering 2012;297:493.

[2] Thunga M, Chen K, Grewell D, Kessler MR. Bio-renewable precursor fibers from lignin/polylactide blends for conversion to carbon fibers. Carbon 2014;68:159.

[3] Gellerstedt G, Sjöholm E, Brodin I. The wood-based biorefinery: A source of carbon fiber? The Open Agriculture Journal 2010;4.

[4] Baker DA, Gallego NC, Baker FS. On the characterization and spinning of an organicpurified lignin toward the manufacture of low-cost carbon fiber. Journal of Applied Polymer Science 2012;124:227.

[5] Kubo S, Kadla J. Lignin-based carbon fibers: Effect of synthetic polymer blending on fiber properties. Journal of Polymers and the Environment 2005;13:97.

[6] Gellerstedt G, Sjöholm E, Brodin I. The wood-based biorefinery: A source of carbon fiber? The Open Agriculture Journal 2010;3:119.

[7] Baker DA, Rials TG. Recent advances in low-cost carbon fiber manufacture from lignin. Journal of Applied Polymer Science 2013;130:713.

[8] Kadla J, Kubo S, Venditti R, Gilbert R, Compere A, Griffith W. Lignin-based carbon fibers for composite fiber applications. Carbon 2002;40:2913.

[9] Ragauskas AJ, Beckham GT, Biddy MJ, Chandra R, Chen F, Davis MF, et al. Lignin valorization: improving lignin processing in the biorefinery. Science 2014;344:1246843.

[10] Luo J, Genco J, Cole BJ, Fort RC. Lignin recovered from the near-neutral hemicellulose extraction process as a precursor for carbon fiber. BioResources 2011;6:4566.

[11] Qiao W, Huda M, Song Y, Yoon S-H, Korai Y, Mochida I, et al. Carbon fibers and films based on biomass resins. Energy \& fuels 2005; 19:2576. 
[12] Prauchner M, Pasa V, Otani S, Otani C. Biopitch-based general purpose carbon fibers: Processing and properties. Carbon 2005;43:591.

[13] Baker FSG, N. C.; Baker, D. A. DOE FY 2008 Progress Report for Lightweighting Materials, Part 7.A., 2008.; 2008.

[14] Wang S, Ru B, Lin H, Sun W, Luo Z. Pyrolysis behaviors of four lignin polymers isolated from the same pine wood. Bioresource technology 2015;182:120.

[15] Wang S, Wang K, Liu Q, Gu Y, Luo Z, Cen K, et al. Comparison of the pyrolysis behavior of lignins from different tree species. Biotechnology Advances 2009;27:562.

[16] Mohan D, Pittman CU, Steele PH. Pyrolysis of wood/biomass for bio-oil: a critical review. Energy \& fuels 2006;20:848.

[17] Rover MR, Hall PH, Johnston PA, Smith RG, Brown RC. Stabilization of bio-oils using low temperature, low pressure hydrogenation. Fuel 2015;153:224.

[18] Hüttermann A, Mai C, Kharazipour A. Modification of lignin for the production of new compounded materials. Applied Microbiology and Biotechnology 2001;55:387.

[19] Effendi A, Gerhauser H, Bridgwater AV. Production of renewable phenolic resins by thermochemical conversion of biomass: a review. Renewable and Sustainable Energy Reviews 2008; 12:2092.

[20] Williams RC, Satrio J, Rover M, Brown RC, Teng S. Utilization of fractionated bio oil in asphalt. 88th annual meeting of the Transportation Research Board, Washington, DC; 2009.

[21] Stevens J, Gardner DJ. Enhancing the fuel value of wood pellets with the addition of lignin. Wood and Fiber Science 2010;42:439.

[22] Qin W, Kadla J. Carbon fibers based on pyrolytic lignin. Journal of Applied Polymer Science 2012;126:E204.

[23] Sahaf A, Laborie M-PG, Englund K, Garcia-Perez M, McDonald AG. Rheological Properties and Tunable Thermoplasticity of Phenolic Rich Fraction of Pyrolysis Bio-Oil. Biomacromolecules 2013;14:1132.

[24] Fratini E, Bonini M, Oasmaa A, Solantausta Y, Teixeira J, Baglioni P. SANS analysis of the microstructural evolution during the aging of pyrolysis oils from biomass. Langmuir 2006;22:306.

[25] Hilten RN, Das K. Comparison of three accelerated aging procedures to assess bio-oil stability. Fuel 2010;89:2741.

[26] Pollard A, Rover M, Brown R. Characterization of bio-oil recovered as stage fractions with unique chemical and physical properties. Journal of Analytical and Applied Pyrolysis 2012;93:129.

[27] Zhou S, Mourant D, Lievens C, Wang Y, Li C-Z, Garcia-Perez M. Effect of sulfuric acid concentration on the yield and properties of the bio-oils obtained from the auger and fast pyrolysis of Douglas Fir. Fuel 2013;104:536.

[28] Meng J, Moore A, Tilotta D, Kelley S, Park S. Toward understanding of bio-oil aging: Accelerated aging of bio-oil fractions. ACS Sustainable Chemistry \& Engineering 2014;2:2011.

[29] Bayerbach R, Nguyen VD, Schurr U, Meier D. Characterization of the water-insoluble fraction from fast pyrolysis liquids (pyrolytic lignin): Part III. Molar mass characteristics by SEC, MALDI-TOF-MS, LDI-TOF-MS, and Py-FIMS. Journal of analytical and applied pyrolysis 2006;77:95.

[30] Wang S, Wang Y, Cai Q, Wang X, Jin H, Luo Z. Multi-step separation of monophenols and pyrolytic lignins from the water-insoluble phase of bio-oil. Separation and Purification Technology 2014;122:248. 
[31] Zhou S, Brown RC, Bai X. The use of calcium hydroxide pretreatment to overcome agglomeration of technical lignin during fast pyrolysis. Green Chemistry 2015;17:4748.

[32] Nordström Y, Norberg I, Sjöholm E, Drougge R. A new softening agent for melt spinning of softwood kraft lignin. Journal of Applied Polymer Science 2013;129:1274.

[33] Yang H, Yan R, Chen H, Lee DH, Zheng C. Characteristics of hemicellulose, cellulose and lignin pyrolysis. Fuel 2007;86:1781.

[34] Bayerbach R, Meier D. Characterization of the water-insoluble fraction from fast pyrolysis liquids (pyrolytic lignin). Part IV: Structure elucidation of oligomeric molecules. Journal of Analytical and Applied Pyrolysis 2009;85:98.

[35] Zhang M, Ogale AA. Carbon Fibers Derived from Acetylated Softwood Kraft Lignin. Polymer Precursor-Derived Carbon: American Chemical Society; 2014, p. 137.

[36] Tagawa T, Miyata T. Size effect on tensile strength of carbon fibers. Materials Science and Engineering: A 1997;238:336.

[37] Ozbek S, Isaac D. Strain-induced density changes in PAN-based carbon fibres. Carbon 2000;38:2007.

[38] Zhang M, Jin J, Ogale AA. Carbon Fibers from UV-Assisted Stabilization of LigninBased Precursors. Fibers 2015;3:184.

[39] Wang S, Lin H, Ru B, Sun W, Wang Y, Luo Z. Comparison of the pyrolysis behavior of pyrolytic lignin and milled wood lignin by using TG-FTIR analysis. Journal of Analytical and Applied Pyrolysis 2014;108:78. 